\title{
What should comparative cognition expect from direct replication studies?
}

Benjamin G. Farrar and Nicola S. Clayton

Department of Psychology, University of Cambridge, Cambridge CB2 3EB, United Kingdom

Corresponding author: bgf22@cam.ac.uk 24/09/2019

An updated version of this pre-print has now been published at Animal Behavior and Cognition. If you are interested in the topic, please see the published manuscript:

Farrar, B. Gr, Boeckle, M., \& Clayton, N. S. (2020). Replications in comparative cognition: What should we expect and how can we improve? Animal Behavior and Cognition, 7(1), 1-22. doi: https://doi.org/10.26451/abc.07.01.02.2020 


\section{Abstract}

What should comparative cognition expect from studies which replicate an experiment's methods as closely as possible? This paper will show that comparative cognition should not expect its just significant findings to replicate consistently, and that published effect sizes are likely overestimated because of a bias towards publishing positive results - a fact that has long been known in other literatures. However, researchers can judge the likely reliability of previous research using a number of features. These include statistical cues, such as p-values and confidence intervals, as well as design cues, such as the control of error variance. All things being equal, studies that use within-subjects designs with many trials and more reliable measurements are more likely to replicate than similar studies that use between-subjects designs, or within-subject designs with few trials or less reliable measurements. However, some areas of comparative cognition will not be able to use designs with many trials, due to methodological or practical constraints. For example, using many trials might invalidate some tests because of learning effects on later trials. Comparative cognition therefore faces replicability-validity and replicability-resource trade-offs, and because of this, researchers should not simply aim for maximum replicability when planning studies. Further, when researchers wish to probe previous results, it will not be possible to perform truly direct replication studies in comparative cognition. This is due to the large degree of temporal, developmental and spatial variation in captive animal behaviour, which means that we should often expect there to be differences between studies using the same methods on different samples. Even when direct replications are performed, sample size constraints mean that these replications will have very uncertain estimates, just as the original studies. As such many claims in the comparative cognition literature may be practically unfalsifiable. Nevertheless, comparative cognition can take steps towards assessing the replicability of its findings, and this will help to re-evaluate the evidential value of the published literature, improve statistical thinking and study design, and identify the many replicable findings that comparative cognition has produced. Future research, whether replication oriented or not, will be more informative if the replicability of comparative cognition is estimated. 


\section{What should comparative cognition expect from direct replication studies?}

Across scientific disciplines there is increasing concern about the reliability and reproducibility of research findings (Baker, 2016). Comparative cognition, however, has yet to have much of an investigation into the reliability of its findings (although several groups are beginning this discussion e.g. Beran, 2018; Bohn et al., 2019; Stevens, 2017). This is surprising given that in psychology and related fields, large-scale attempts to replicate published research have only replicated significant results around 60\% of the time (Camerer et al., 2016, 2018; Ebersole et al., 2016; Klein et al., 2018; Open Science Collaboration, 2015; Zwaan et al., 2018). However, the generalizability of these findings to comparative cognition are uncertain because of the substantial variation in replicability between research questions and disciplines. Nevertheless, in fields similar to comparative cognition, namely infant cognition and animal learning, some established effects do not consistently replicate (e.g. Maes et al., 2016; Poulin-Dubois et al., 2018).

It should be noted, however, that comparative cognition is a heterogenous field: some areas of comparative cognition do bear the hallmarks of science that has proven difficult to reproduce namely small sample sizes, noisy measurements and unlikely hypotheses (Forstmeier, Wagenmakers, \& Parker, 2017); whereas other areas might be less affected by low replicability rates due to using research designs which typically show higher replicability, such as within-subjects designs in which there are many trials for each animal tested and a strong contact between data and quantitative theoretical predictions (Smith \& Little, 2018).

In light of this, what should comparative cognition expect from direct replication studies? This paper aims to answer this question in relation to studies that examine behaviour at the group-level, and focuses on studies where there might be some doubt about the replicability of the effect, i.e. where the strength of evidence in the original study is not overwhelming. Importantly, comparative cognition will have produced many robust and reliable effects, and the contents of this paper are not equally applicable across every research question and design: however, it provides suggestions as to how to identify studies or research programmes that might not contain reliable results.

Section 1 of this paper will examine statistical cues to replicability, and specifically ask what can be gained from looking at p-values and effect sizes in published findings. Section 2 then asks whether the different research designs employed across comparative cognition may lead to systematic differences in the replicability of results. Next, Section 3 discusses the difficulties that comparative cognition will face when interpreting the results of replication studies, and finally Section 4 will make the case that, even if the results of negative replication studies may be near uninterpretable with respect to each 
individual claim, they will be valuable in understanding and developing comparative cognition as a science.

\section{Section 1 - p-values, effect sizes and direct replications}

Before examining comparative cognition studies themselves, cues to replicability can be found in the statistical reports of experiments. Perhaps counter-intuitively, when a published experiment with a significant result is directly replicated, it can be more likely than not that the direct replication study will not return a significant result in the same direction as the original study. This is even the case when the original study produced a true positive finding. To see why this is the case, consider three scenarios of a direct replication study, in which the original study estimated the unknown true effect size exactly, underestimated it or overestimated it. These direct replication studies have the same sample size as the original study and sample from the same population.

\section{Scenario 1 -original study estimated the real effect size exactly}

If an original study estimated the real effect size for that design accurately, then the likelihood of the replication study to return a positive result is the power of the statistical test to detect this effect size. If the original study returned a positive result that was just statistically significant, e.g. $p=.049$, the likelihood of an exact replication study returning a positive result is approximately $50 \%$; like tossing a coin (Piper et al., 2019). This is because, $50 \%$ of the time the replication study will overestimate the effect size, resulting in $p<.049$, whereas $50 \%$ of the time it will underestimate the effect size, resulting in $p>.049$, i.e. non-significant with an alpha of .05 . However, as the $p$-value of the original study decreases, then ceteris paribus, the chances of a positive replication result increase.

\section{Scenario 2 - original study underestimated the real effect size}

If the original study underestimated the real effect size, then it becomes more likely that a direct replication study of equal sample size will return a positive result, as the replication study will exceed that effect size over $50 \%$ of the time. In the example of a just significant result, $p=.049$, in the original study, the likelihood of a positive direct replication with the same sample size is now greater than $50 \%$.

Scenario 3 - original study overestimated the real effect size

However, the most likely scenario is that the original study will have overestimated the real effect size, particularly for studies that produced $p$ values just below the alpha level. This scenario is most likely for two reasons. First, as publications are selected based on a cut-off point, studies that overestimate effect sizes are more likely to be published than studies which underestimate effect sizes. For example, 
if exactly estimating the "true" effect size would have yielded a p-value of .06 in a design, then only samples overestimating the effect size would be published. Hence, in direction replication studies, the replication $p$ value would regress to the mean (.06), and the original study would have less than a $50 \%$ chance of a positive direct replication with the same sample size (Fiedler \& Prager, 2018; Sterling, 1959). Second, the overestimation of published effect sizes is further exacerbated if research practices that produce more significant results are used, which appears relatively common (Fraser, Parker, Nakagawa, Barnett, \& Fidler, 2018; John, Loewenstein, \& Prelec, 2012; Simmons, Nelson, \& Simonsohn, 2011). Hence, the default expectation when directly replicating a study with a $p$ value in the range of $.01<p<.05$ is that there is a good possibility that a direct replication study with the same sample size will not produce a statistically significant result in the same direction as the original study.

\section{Section 1.1 - Simulation study}

The fickleness of $p$-values, and consequences of having a statistical significant filter has long been known to produce results that are inconsistent (Cohen, 1990; Cumming, 2008) and effect sizes that are overestimated (Hedges, 1984), which is particularly the case in areas in which measurement error is high (Gelman \& Carlin, 2014; Loken \& Gelman, 2017). These ideas are easiest to see through simulation studies, and to this end we simulated a very simple model of comparative cognition research (For details see Appendix A) In this, 40,000 studies were simulated, comprising of four sets of 10,000 studies with a power of $80 \%, 50 \%, 20 \%$ and $5 \%$ (i.e. no effect) to detect a known difference between two groups of 10 animals. The groups were compared using a one-tailed two-sample t-test and results with $p<.05$ were termed significant and "published". The results of these studies are displayed in Table 1, along with a comparison of the actual and published effect sizes.

Table 1: The results of 40,000 simulated comparative cognition studies by power. The
proportion published represents the proportion of studies producing $\mathrm{p}<.05$, and the
unstandardised effect sizes are the mean differences between the groups.
$\begin{array}{ccccc}\text { Proportion } & \text { Unstandardised Effect Size } \\ \text { Power } & \text { Published } & \text { All Samples } & \text { Published } & \text { Inflation } \\ 80 & 0.796 & 5.77 & 6.53 & 13 \% \\ 50 & 0.494 & 3.82 & 5.56 & 45 \% \\ 20 & 0.205 & 1.89 & 4.88 & 158 \% \\ 5 & 0.053 & 0.007 & 4.45 & 64486 \%\end{array}$


Even when running studies with $80 \%$ power, the average effect size is inflated if only the significant studies are published. As the power of the tests decrease, the degree of inflation increases. Next, we derived the expected proportion of successful ${ }^{1}$ direct replication studies from the 15,471 published studies. The direct replications had equal sample size to the original studies, and the results are broken down across different ranges of the published $p$ values in Table 2. The overall expected replication rate of this group of research, conducted with a quarter of studies having $80 \%, 50 \%, 20 \%$ and $5 \%$ power was 0.62 .

Table 2: The mathematically derived probability of a successful replication attempt of an original study randomly selected from a given range of p-values from the 15,471 "published" simulation studies.

$\begin{array}{cccccc}\text { P-value original study } & p \leq 0.01 & 0.01<p \leq 0.02 & 0.02<p \leq 0.03 & 0.03<p \leq 0.04 & 0.04<p \leq 0.05 \\ \begin{array}{c}\text { Probability of } \\ \text { successful replication }\end{array} & 0.67 & 0.57 & 0.52 & 0.50 & 0.48\end{array}$

These results show that the expected replication rate of published research from this model can be close to 0.5 , or even below 0.5 , for just significant results. Notably, these results are in the absence of any false positive inflating research practices (Fraser et al., 2018; John et al., 2012; Simmons et al., 2011); they are solely the consequence of only publishing research with $p<0.05$ and running at least some studies with relatively low power.

\section{Conclusion 1: Comparative cognition should not expect just significant initial findings to consistently replicate, and published effect sizes are likely to be overestimated because of a publication bias towards positive results}

However, this simulation does not accurately characterise the field of comparative cognition because not all of the research is performed with $80 \%, 50 \%, 20 \%$ or $5 \%$ power, or using two-sample t-tests comparing two groups of 10 animals. As such the numbers should not be taken as a literal estimate of the replicability of comparative cognition research; rather the simulation study shows that the research and publication methods used in comparative cognition lead to effect sizes being over-

\footnotetext{
${ }^{1}$ Replications were "successful" if they returned a significant result in the same direction as the original study. Terming a replication successful or not is ultimately not a fruitful way of conceptualizing replications, which will be discussed later.
} 
estimated and a replication success likely to be closer to $50 \%$ than $100 \%$ for just significant published findings ${ }^{2}$.

Section 2 now focuses on whether the differences in research methods across comparative cognition, that were not considered in the simulation study, can allow us to make predictions about whether certain areas of comparative cognition might be more susceptible to replicability issues than others.

\section{Section 2 - Replications across comparative cognition: What can we expect?}

Section 1 showed that, all else being equal, studies in comparative cognition with lower $p$-values are more likely to replicate than studies with higher $p$-values, although the $p$ value is only a weak predictor of replication success (Cumming, 2008). However, this begs the question: which features of comparative cognition research lead to effect sizes large enough to be reliably detected, and thus produce low $p$-values and successful replication studies? ${ }^{3}$

One of areas in which comparative cognition research methods differ is in their ability to control error variance. Given that sample sizes are often small in comparative cognition, many "effects" might be too small to detect on a single trial, as the errors associated with measuring these behaviours on a single trial are large. Hence, studies will often use within-subjects designs, in which many trials are performed by each individual animal. This enables more precise estimation and larger effect sizes, and consequently smaller $p$-values and more replicable results to manifest. This tendency of within-subject design with many trials to be more replicable is visible in the human replication literature (Open Science Collaboration, 2015; Zwaan et al., 2018), and this is due to within-subject designs being more powerful to detect effects than between-subject designs with similar resources, i.e. a higher signal to noise ratio. Many research programmes in comparative cognition harness this ability to perform repeated tests on individual animals that are often highly trained at the task, although whether a

\footnotetext{
${ }^{2}$ One scenario in which these conclusions might be inappropriate is considering research using non-parametric tests and small sample sizes, in which the $p$-value distribution is not uniform or near uniform under the null hypothesis - however this extreme condition likely only applies to a very small proportion of all published research.

${ }^{3}$ While this paper focuses on statistical significance, as this is the framework that most research is performed in, the quality of statistical and scientific thinking of researchers might be improved by moving beyond significance and focusing on accepting and communicating uncertainty (Gelman, 2017; Gelman \& Carlin, 2017; McShane \& Gal, 2016; McShane, Gal, Gelman, Robert, \& Tackett, 2019).
} 
certain number of trials are "sufficient" or not will depend on particular features of the task at hand, notably the size of the effect and the measurement error ${ }^{4}$.

Conclusion 2: Studies using within-subject designs with many trials and less-noisy behaviours are more likely to produce replicable results than studies with similar resources that use betweensubjects designs, within-subject designs with few trials or more-noisy behaviours

However, this benefit of running many trials across participants may not be accessible for some areas of comparative cognition. This is the case when theoretical constraints restrict experimental designs to using a single test trial, in order to avoid experimental confounds. For example, in detour tests with visible rewards, performance is often analysed on the first trial as a test of "insight" rather than a test of learning abilities (Kabadayi, Bobrowicz, \& Osvath, 2018). In some memory tests the subjects are tested in extinction, to prevent cues from the to-be-remembered items directly influencing their searching or responding behaviour (Adams \& Dickinson, 1981; Clayton \& Dickinson, 1998). The point being made here is that such studies cannot use many test trials in within-subjects designs because this would invalidate the test itself because the animals could have learned that no food is provided in the test trials and therefore that there is no value in searching for the food. For some hypotheses in comparative cognition, the optimal methodological design may not be one that produces highly replicable results.

Thus far, we have presented within-subjects designs as varying continuously on one dimension, the number of trials, and have suggested that all else equal, many trials are better than few. However, this does not mean that most many trial studies and no few trial studies will replicate successfully. Further, even if this were true, it does mean that many trial studies are always better. As ever, there are trade-offs between likely replicability, validity and resource investment. Many experiments that use many trials, such as reaction time studies in humans, can have participants perform tens of trials in minutes, whereas in animal studies this is not possible. The reliability-resource trade-off is just that: a trade-off, and researchers should seek to evaluate and improve reliability with this in mind.

Conclusion 3: Some areas of comparative cognition face replicability/validity and replicability/resource trade-offs

\footnotetext{
${ }^{4}$ Researchers wishing to plan studies with this in mind could conduct design sensitivity analyses (see Morey, 2019; R. Morey \& Lakens, 2016) or design analyses (Gelman \& Carlin, 2014), which provide much more information than conventional power analyses.
} 


\section{Section 2.1 - Replications across comparative cognition: Small-N, Many Replicates?}

In contrast to the low signal-to-noise ratio found in much research, there are some areas of psychology, comparative cognition included, that may be relatively robust from issues with replicability. One of these areas use Small-N designs (Little \& Smith, 2018; Smith \& Little, 2018). Small$\mathrm{N}$ designs capitalise on the benefits of using many trials on each individual subject, yet rather than seek to estimate group-level parameters they treat the individual as the replication unit. Each individual is its own experiment, and experiments are thus replicated by using more than one individual. Smith and Little explain this approach:

"We argue that some of the most robust, valuable, and enduring findings in psychology were obtained, not using statistical inference on large samples, but using small-N designs in which a large number of observations are made on a relatively small number of experimental participants. We argue that, if psychology is to be a mature quantitative science, its primary theoretical aim should be to investigate systematic, functional relationships as they are manifested at the individual participant level. The estimation of population parameters, while not unimportant, is arguably of secondary concern and should probably be investigated using more refined techniques for characterizing individual differences than the blunt instrument of simple averaging that conventional statistical methods provide."

Smith \& Little, (2018, pg. 2084)

The small-N approach is found across psychological research, for example in Ebbinghaus' forgetting curve, many psychophysics experiments and some animal learning studies. It is true that interesting hypotheses in comparative cognition are often found at the individual level, particularly when there are strong a priori reasons that results at the individual level will generalise at least some more individuals. For example, understanding the learning mechanisms of Lab Rat 377's learning logically will inform us about rat learning more generally, hence a small-N approach might be appropriate here. However, while small- $\mathrm{N}$ research has a formal approach to inference at the individual level (Smith \& Little, 2018), there is no formal method of generalizability. In comparative cognition, when strong evidence is found at the individual level $l^{5}$, the question "will it replicate?" is synonymous to "will it generalise?". In contrast, in most comparative cognition research, when evidence is provided at the group level, the question "will it replicate?" may be more synonymous to "is it true?".

\footnotetext{
${ }^{5}$ This evidence can be very convincing as existence proof. For example, when Megan passed the trap tube task (Povinelli, 2003), this was overwhelming evidence that chimpanzees are in theory capable of learning how to pass the task. Note, this evidence is only overwhelming because of how inconsistent Megan's behaviour was with her not avoiding the trap, i.e. a p-value of 0.000000000135 (80/100 correct trials, with a null hypothesis as $50 / 100)$.
} 
There may be benefits in comparative cognition moving from the currently dominant large- $\mathrm{N}$ approach towards a small- $\mathrm{N}$ approach, particularly in areas in which there might be reliability concerns. If these experiments focused on performing highly-powered tests at the individual level, they could get more certain estimates of whether each individual animal displays an effect, and be confident in the robustness of this inference. If all animals displayed the effect, the inference that it is a general phenomenon would be clearly warranted. In contrast, if only some animals displayed the effect, with a high degree of confidence, this would be an interesting finding in itself - and one that would have been missed with a conventional approach. ${ }^{6}$

Conclusion 4: Different research approaches have different meanings by replicability (see Lazic, Clarke-Williams, \& Munafò, 2018). These approaches answer different questions, and comparative cognition would benefit from exploring whether its questions are best answered at the individual or population level.

\section{Section 2.2 - The data are not everything}

So far, this paper has considered statistical and design features of replicability. However, there is much more information about replicaibility than can be gleaned from reported statistics alone. When Daryl Bem provided evidence for precognition, it is true that statistical markers were part of the subsequent refutation (Francis, 2012; Schimmack, 2012; Wagenmakers, Wetzels, Borsboom, \& van der Maas, 2011). However, it was the sheer implausibility of the effect that provided the clearest indicator that it would not replicate (Chambers, 2017). This ability of researchers to detect likely unreliable findings extends from the physically impossible to more plausible research results too: research using forecasting and prediction markets has shown that experts can be surprisingly accurate at identifying research that will not replicate (Dreber et al., 2015; Forsell et al., 2018)- and there is no reason why this should be any different in comparative cognition. If your belief in something is very low, then a just-significant result should not affect this belief greatly.

Conclusion 5: The data aren't the full story - beliefs about replicability might be accurate, whatever they are based on

\footnotetext{
${ }^{6}$ Such data could also be analysed with mixed-effects models if researchers still wanted to estimate population parameters - i.e. the benefits of many trials is compatible with both individual and population level inferences.
} 
Section 3 - Difficulty in performing and interpreting replication studies in comparative cognition

Given that many research findings might be unreliable in comparative cognition, a next step could be to call for a suite of systematic, direct replications studies to identify findings that are robust. While there are strong reasons to support such claims (e.g. see Beran, 2018 and Stevens, 2017, although neither make such a claim), there are many barriers to performing and interpreting replication studies in comparative cognition that should be considered. First and foremost, it may not be possible to even attempt to directly replicate some studies, and second, even when studies are directly replicated, negative results will be unlikely to provide convincing evidence against a claim. Because of this that some areas of comparative cognition might contain results that are practically unfalsifiable.

It is not possible to attempt to directly replicate some findings

As comparative cognition is a small field represented by many different research questions in many different species (Beran, Parrish, Perdue, \& Washburn, 2014; Shettleworth, 2009), it is possible that when a laboratory stops working on a certain species, the likelihood of directly replication studies of these results in the near future approaches zero.

For example, our Comparative Cognition laboratory in Cambridge performed a series of studies on cache-protection strategies in California scrub-jays (Aphelocoma californica), which it no longer houses ${ }^{7}$. Consequently, the possibility of these studies being directly replicated on California scrubjays in this lab in the coming years is extremely unlikely. However, in light this, what confidence should we have in the published research? This should vary from effect to effect, depending on the strength of evidence for each claim. For example, for claims that have been replicated relatively directly several times the reduction in belief about whether this is a real effect or not should only be small ${ }^{8}$. More caution should be given to results supported by a single study, for example the claim that California

\footnotetext{
${ }^{7}$ This was because the existing birds died and it has not been possible to obtain new birds for various practical reasons: the birds need to be acquired as chicks from nests in the wild and hand-raised in California, which requires personnel and lab facilities in the USA; a suite of export and import permits; transport to the UK and housing in quarantine facilities, which are no longer available in Cambridge. Assuming ng all the birds survive and are therefore not euthanized because of legal and disease issues, they can then finally be housed and tested in Cambridge. In contrast, instead labs can turn to native species, as in the Clayton lab, which now houses Eurasian jays (Garrulus glandarius).

${ }^{8}$ For example, the finding that scrub-jays re-cache a higher proportion of food items that were cached under observation by a conspecific, than when they were cached in private in-private, was replicated relatively directly eight times across four publications (Dally, Emery, \& Clayton, 2006; Emery \& Clayton, 2001; Emery, Dally, \& Clayton, 2004; Thom \& Clayton, 2013).
} 
scrub-jays match caching items to the colour of the caching substrate, since this study has only been conducted once and produced a critical $p$ value of .03 (Kelley \& Clayton, 2017).

In saying that we should reduce confidence in some findings we are not referring to the absolute truth of the claim. It may be almost certain that on average, across all caching events ever, items are matched to the colour of the substrate. However, the claim implicit to Kelley and Clayton is not that this effect exists, i.e. that it was correct to reject the null hypothesis, but the suggestion is that this effect would be large enough to be found in study designs similar to their own, i.e. that the original study did not commit a Type M error (Gelman \& Carlin, 2014).

\section{Conclusion 6: Often, direct replications will not be possible, but the reliability of this research should still be examined}

However, in contrast to the occasional instances when certain species are no longer available, it will be possible to attempt direct replication studies for many research findings. However, next we argue that these results may not be overly informative, aside from in highlighting the large degree of uncertainty already present in our research findings. In particular, negative results from such replication studies will be near uninterpretable because i) the small sample sizes attainable in comparative cognition make it near impossible to detect a difference between original and replication results, and ii) site and time differences between studies means that many results should not be expected to replicate consistently.

\section{Small sample sizes}

When the Open Science Collaboration (OSC) replicated 97 studies with a positive effect, only 36 of these produced a statistically significant result in the same direction as the original study (Open Science Collaboration, 2015). However, considering these significant replications to be the only "successful" studies might be misleading (Gelman, 2018). This is because it dichotomises replication attempts as either successful $(p<0.05)$, or unsuccessful $(p>0.05)$. While a replication study with $p=$ .049 would be considered a success, a replication study with $p=.051$ would be considered a failure. ${ }^{10}$ However, as the average sample size in the OSC replication study was not greatly larger than the original studies, it is not surprising that many replication studies did not yield significant results (see

\footnotetext{
${ }^{9}$ This is not to say that we believe the result to be a false positive, rather we believe that it is far from certain that if the study was replicated exactly it would return a significant result in the same direction as the original study.

${ }^{10}$ And this criticism equally applies to the "probability of successful replication" reported in the simulation study (Section 1).
} 
Section 1). In fact, more liberal, and perhaps more appropriate, interpretations of the Open Science Collaboration results provide higher estimates of replicability. For example, Etz and Vandekerckhove (2016) used a Bayesian analysis to suggest that " $75 \%$ of [replication] studies gave qualitatively similar results [to the original studies]", but also noted that "the majority of the studies (64\%) did not provide strong evidence for either the null or the alternative hypothesis in either the original or the replication". Future replication projects in human psychology have attempted to address this concern by swamping the sample sizes in the original studies (e.g. Camerer et al., 2018). However, as Morey and Lakens note (Morey \& Lakens, 2016), "sample sizes are so small in psychology that often one cannot detect even large differences between studies. High-powered replications cannot answer this problem, because the power to find differences in results from a previous study is limited by the sample size in the original study". When this concern is translated to comparative cognition, and the constraints on sample sizes which it faces, it is clear that producing meaningful replication studies that are capable of assessing the veracity of the original claim, will be very challenging.

Conclusion 7: Comparative cognition might not have the resources to produce highly-powered and informative replication studies, and as such many claims are practically unfalsifiable

\section{Temporal variation in performance within animals and groups}

However, assuming some form of direct replication studies are performed, interpreting the results becomes even more difficult when the many reasons why a comparative cognition replication study might fail to produce significant results are considered. One example is that animal behaviour often has temporal variation: a direct replication study of caching behaviour in Eurasian jays performed in the UK in August would fail to replicate results of an original study performed in December, because in August the birds do not cache much, if at all. In addition to these consistent between-season differences in animal behaviour, there are also systematic changes in animal behaviour across their lifespans. As many studies in comparative cognition are performed on the same individuals, how these individuals change over their lifespan will affect whether results should replicate or not. For example, a memory experiment performed on young animals might not replicate when the same animals are used in their old age (e.g. Robitsek, Fortin, Koh, Gallagher, \& Eichenbaum, 2008). This could either be due to the original results being a false positive, or the well-motivated alternative hypothesis that the memory performance of the animals decreases with age.

Conclusion 8: Temporal and developmental variation in animal behaviour will influence the likelihood of replication success 


\section{Differences in performance between sites between animals}

Needless to say, when direct replication studies using a new sample from the same population can be performed, these will be an effective method of assessing the validity of an effect. However, in comparative cognition, it is not feasible to assume new samples can be repeatedly taken from the same population - in fact it is unclear what "populations" we are studying in general. Rather, different groups of animals of the same species from different research sites may be best viewed as different populations with respect to most cognitive effects.

In biomedical research, researchers see differences in physiology and behaviour between laboratories, even when they test the exact same strain of animals with the exact same protocol (Crabbe, Wahlsten, \& Dudek, 1999). These real differences in nominally similar animals when exposed to the same treatment may reduce expectations of between-laboratory replicability (VoelkI \& Würbel, 2016) ${ }^{11}$, and within-laboratory replicability when the same animals are tested repeatedly (Karp et al., 2014). That these differences are present in such standardised conditions raises the likelihood that real and potentially large effects of site and time will reduce the replicability of animal cognition research.

A recent example in animal cognition comes from the ManyPrimates collaboration. Here, they collected data on 176 individual primates from 12 species on a delayed response task, in which primates had to wait either 0,15 or 30 seconds before choosing the location where food was hidden (Bohn et al., 2019). While there are notable similarities within and between species behaviour across sites, some large differences can still be observed ${ }^{12}$. For example, the 12 chimpanzees from the Wolfgang Köhler Primate Research Center greatly outperformed the 12 chimpanzees from Edinburgh Zoo. If such a between site difference is present in a relatively simple and robust cognitive task, in which inter-individual differences might be expected to be low (Hedge, Powell, \& Sumner, 2018), this suggests that even larger between site differences will manifest for more noisy behaviours in comparative cognition replication studies. A further implication of this is that multi-site studies examining between species behaviour may be confounded to a certain extent precisely because they

\footnotetext{
${ }^{11}$ These differences in behaviour might reflect an adaptive response to many subtle environmental differences between sites, and as such "independent replicate studies that fail to reproduce the original findings might not necessarily indicate that the original study was poorly done or reported, but rather that the replicate study was probing a different region of the norm of reaction."(Voelkl \& Würbel, 2019). The reaction norm is a concept used by Voelkl and Würbel to represent gene $x$ environment interactions acknowledging both plasticity and canalisation.

${ }^{12}$ Although these large differences were observed by looking at the data to find such an example, it seems like this difference is large and real.
} 
are multi-site, which could make the results of species represented by single sites difficult to interpret. Perhaps then, at best, direct replications between sites in comparative cognition should be seen as equivalent to cross-cultural studies in humans, and as such lie closer to conceptual rather than direct replications.

Conclusion 9: Site specific differences in behaviours make direct replication studies sampling from new populations difficult to interpret, and could confound many between species comparisons

It's all about the effect size

However, while site or time differences been studies will modulate effects, this does not mean that they should determine whether an effect should be present in one study and entirely absent in another: The effect of ageing on animals should not completely remove their ability to pass memory tests, and similarly group differences in chimpanzee cognition should modulate the size of the effect of working memory in a delayed-response task, not the presence of it. Instead of focussing on the "presence" or "absence" of an effect in a replication study a more fruitful approach would be to look for differences between original studies and their replications (Gelman, 2018; R. Morey \& Lakens, 2016).

If replications are highly powered, they will nearly always be different to original studies because of site-specific differences. Hence, rather than just looking for a difference between original and replication finding, researchers should ask is the replication result meaningfully different from the original study, and how does this affect the theoretical conclusions of the research. What a theoretically meaningful difference between original study and replication study, or between effect and no effect, is a difficult concept to approach, but one that is necessary to consider if comparative cognition is to gain much insight from replication studies. Unfortunately, reaching sufficient power to detect meaningful effects or differences between studies might be unattainable for many current designs comparative cognition. However, this does not prevent the possibility that performing replication studies within the current constraints will produce many near-incomprehensible results, precisely because they are not powered to detect meaningful differences between studies.

Conclusion 10: A focus on effect sizes, and meaningful differences between studies, should be a long term, but this is a currently unrealistic aim for many effects in comparative cognition. 


\section{Section 4 - What comparative cognition can learn from direct replication studies}

While the results of replication studies might be difficult to interpret, we still believe that performing such replication will still be useful in identifying reliable and robust effects in comparative cognition and calibrating researcher expectations for future research.

A major benefit of replication studies in comparative cognition is that, when pre-registered (Nosek, Ebersole, DeHaven, \& Mellor, 2018) replication studies do successfully replicate results, "real" effects can be identified with confidence. These effects can then provide a firm foundation for future research. In contrast, if pre-registered replications do not reliably replicate then the effects themselves warrant further investigation, rather than being a platform on which to build future research. Thus, by identifying effects which are robust and effects which are weak, direct replication studies can prevent future resource waste. Further, the results of replication studies generalise. If a result is not easily replicable in comparative cognition, then it also suggests that similar effects from similar designs will be difficult to replicate - or to produce in the future. If a new research programme is designed to extend on previous findings, information about the replicability of these previous findings is essential to understand the future results. Replication studies, even those lacking in power, can increase the informativeness of research that has not yet been performed.

Further, the results of replication studies in comparative cognition can suggest ways to improve its scientific method. For example, if site-specific differences do appear to routinely modulate effects, then studies could systematically vary the conditions and apparatus they use within experiments. Such a design attempts to control for between site variability in nominally irrelevant factors, allowing an overall effect to be estimated that might be more robust to site-specific differences (Baribault et al., 2018; see also Voelkl, Vogt, Sena, \& Würbel, 2018). If researchers anticipate constrains on the generalisability of their results, these well-motivated a priori reasons could be stated with the original finding (Simons, Shoda, \& Lindsay, 2017). As almost any negative replication could be explained by a combination of post-hoc reasons, this would protect the strongest reasons from being attributed to post-hoc reasoning.

Finally, comparative cognition is not alone in facing issues with, and confusion about, replicability. Other fields which have begun to address these concerns are seeing a "credibility revolution" (Vazire, 2018). One fruitful approach to this has been through large multi-site collaborations, and excitingly animal cognition research is trialing infrastructures that could support this, such as ManyPrimates (Bohn et al., 2019) and the planned ManyDogs. Irrespective of whether these collaborations choose to pursue replication studies, or purely novel research, they have the potential to solve some of the core issues relating to statistical power and study planning that comparative cognition currently faces 
- one of the most important questions for these collaborations is to identify the most important questions on which to focus their resources.

\section{Concluding Remarks}

Often direct replication studies of comparative cognition research will be difficult to perform, and even more difficult to interpret. Many features of the "credibility revolution" in other disciplines will be unavailable to comparative cognition researchers, who cannot access large and diverse samples. However, these concerns do not apply equally throughout comparative cognition - those working with more easily accessible animals, such as dogs, pigeons or rats, might be able to run informative replication studies and for other species collaboration between labs will be key. For this reason, largescale collaborations such as ManyPrimates, are poised to offer a new quality of evidence for comparative cognition studies on hard to reach species.

Although comparative cognition faces problems in interpreting the reliability of many of its published findings, these problems present an opportunity to understand what evidence comparative cognition has produced and is capable of producing. Future research, whether replication oriented or not, will be more informative if the replicability of comparative cognition is estimated.

\section{Acknowledgements}

We would like to thank Ljerka Ostojić and Rachel Crosby, who's discussions on this topic have been extremely helpful in forming the arguments presented in the paper. 


\section{References}

Adams, C. D., \& Dickinson, A. (1981). Instrumental Responding following Reinforcer Devaluation. The Quarterly Journal of Experimental Psychology Section B, 33(2b), 109-121.

https://doi.org/10.1080/14640748108400816

Baker, M. (2016). 1,500 scientists lift the lid on reproducibility. Nature News, 533(7604), 452. https://doi.org/10.1038/533452a

Baribault, B., Donkin, C., Little, D. R., Trueblood, J. S., Oravecz, Z., van Ravenzwaaij, D., ... Vandekerckhove, J. (2018). Metastudies for robust tests of theory. Proceedings of the National Academy of Sciences, 115(11), 2607-2612.

https://doi.org/10.1073/pnas.1708285114

Beran, M. J. (2018). Replication and Pre-Registration in Comparative Psychology. Retrieved from https://escholarship.org/uc/item/59f4z2nd

Beran, M. J., Parrish, A. E., Perdue, B. M., \& Washburn, D. A. (2014). Comparative Cognition: Past, Present, and Future. International Journal of Comparative Psychology / ISCP ; Sponsored by the International Society for Comparative Psychology and the University of Calabria, 27(1), $3-30$.

Bohn, M., Schmitt, V., Sanchez-Amaro, A., Keupp, S., Hopper, L., Völter, C., ... Hernandez-Aguilar, R. A. (2019). Establishing an infrastructure for collaboration in primate cognition research [Preprint]. https://doi.org/10.31234/osf.io/3xu7q

Camerer, C. F., Dreber, A., Forsell, E., Ho, T.-H., Huber, J., Johannesson, M., ... Wu, H. (2016). Evaluating replicability of laboratory experiments in economics. Science, 351(6280), 14331436. https://doi.org/10.1126/science.aaf0918

Camerer, C. F., Dreber, A., Holzmeister, F., Ho, T.-H., Huber, J., Johannesson, M., ... Wu, H. (2018). Evaluating the replicability of social science experiments in Nature and Science between 2010 and 2015. Nature Human Behaviour, 2(9), 637. https://doi.org/10.1038/s41562-0180399-z 
Chambers, C. (2017). The seven deadly sins of psychology: A manifesto for reforming the culture of scientific practice / Chris Chambers. Princeton: Princeton University Press.

Clayton, N. S., \& Dickinson, A. (1998). Episodic-like memory during cache recovery by scrub jays. Nature, 395(6699), 272-274. https://doi.org/10.1038/26216

Cohen, J. (1990). Things I have learned (so far). American Psychologist, 45(12), 1304-1312. https://doi.org/10.1037/0003-066X.45.12.1304

Crabbe, J. C., Wahlsten, D., \& Dudek, B. C. (1999). Genetics of Mouse Behavior: Interactions with Laboratory Environment. Science, 284(5420), 1670-1672. https://doi.org/10.1126/science.284.5420.1670

Cumming, G. (2008). Replication and p Intervals: P Values Predict the Future Only Vaguely, but Confidence Intervals Do Much Better. Perspectives on Psychological Science, 3(4), 286-300. https://doi.org/10.1111/j.1745-6924.2008.00079.x

Dally, J. M., Emery, N. J., \& Clayton, N. S. (2006). Food-caching western scrub-jays keep track of who was watching when. Science (New York, N.Y.), 312(5780), 1662-1665. https://doi.org/10.1126/science.1126539

Dreber, A., Pfeiffer, T., Almenberg, J., Isaksson, S., Wilson, B., Chen, Y., ... Johannesson, M. (2015). Using prediction markets to estimate the reproducibility of scientific research. Proceedings of the National Academy of Sciences, 112(50), 15343-15347. https://doi.org/10.1073/pnas.1516179112

Ebersole, C. R., Atherton, O. E., Belanger, A. L., Skulborstad, H. M., Allen, J. M., Banks, J. B., ... Nosek, B. A. (2016). Many Labs 3: Evaluating participant pool quality across the academic semester via replication. Journal of Experimental Social Psychology, 67, 68-82. https://doi.org/10.1016/j.jesp.2015.10.012

Emery, N. J., \& Clayton, N. S. (2001). Effects of experience and social context on prospective caching strategies by scrub jays. Nature, 414(6862), 443-446. https://doi.org/10.1038/35106560 
Emery, Nathan J., Dally, J. M., \& Clayton, N. S. (2004). Western scrub-jays ( Aphelocoma californica ) use cognitive strategies to protect their caches from thieving conspecifics. Animal Cognition, 7(1), 37-43. https://doi.org/10.1007/s10071-003-0178-7

Etz, A., \& Vandekerckhove, J. (2016). A Bayesian Perspective on the Reproducibility Project: Psychology. PLOS ONE, 11(2), e0149794. https://doi.org/10.1371/journal.pone.0149794

Fiedler, K., \& Prager, J. (2018). The Regression Trap and Other Pitfalls of Replication ScienceIllustrated by the Report of the Open Science Collaboration. Basic and Applied Social Psychology, 40(3), 115-124. https://doi.org/10.1080/01973533.2017.1421953

Forsell, E., Viganola, D., Pfeiffer, T., Almenberg, J., Wilson, B., Chen, Y., ... Dreber, A. (2018). Predicting replication outcomes in the Many Labs 2 study. Journal of Economic Psychology, S0167487018303283. https://doi.org/10.1016/j.joep.2018.10.009

Forstmeier, W., Wagenmakers, E.-J., \& Parker, T. H. (2017). Detecting and avoiding likely falsepositive findings-A practical guide. Biological Reviews of the Cambridge Philosophical Society, 92(4), 1941-1968. https://doi.org/10.1111/brv.12315

Francis, G. (2012). Too good to be true: Publication bias in two prominent studies from experimental psychology. Psychonomic Bulletin \& Review, 19(2), 151-156.

https://doi.org/10.3758/s13423-012-0227-9

Fraser, H., Parker, T., Nakagawa, S., Barnett, A., \& Fidler, F. (2018). Questionable research practices in ecology and evolution. PLOS ONE, 13(7), e0200303. https://doi.org/10.1371/journal.pone.0200303

Gelman, A. (2017). Ethics and Statistics: Honesty and Transparency Are Not Enough. CHANCE, 30(1), 37-39. https://doi.org/10.1080/09332480.2017.1302720

Gelman, A. (2018). Don't characterize replications as successes or failures. Behavioral and Brain Sciences, 41. https://doi.org/10.1017/S0140525X18000638

Gelman, A., \& Carlin, J. (2014). Beyond Power Calculations. Perspectives on Psychological Science, 9(6), 641-651. https://doi.org/10.1177/1745691614551642 
Gelman, A., \& Carlin, J. (2017). Some Natural Solutions to the $p$-Value Communication ProblemAnd Why They Won't Work. Journal of the American Statistical Association, 112(519), 899901. https://doi.org/10.1080/01621459.2017.1311263

Hedge, C., Powell, G., \& Sumner, P. (2018). The reliability paradox: Why robust cognitive tasks do not produce reliable individual differences. Behavior Research Methods, 50(3), 1166-1186. https://doi.org/10.3758/s13428-017-0935-1

Hedges, L. V. (1984). Estimation of Effect Size under Nonrandom Sampling: The Effects of Censoring Studies Yielding Statistically Insignificant Mean Differences. Journal of Educational Statistics, 9(1), 61. https://doi.org/10.2307/1164832

John, L. K., Loewenstein, G., \& Prelec, D. (2012). Measuring the Prevalence of Questionable Research Practices With Incentives for Truth Telling. Psychological Science, 23(5), 524-532. https://doi.org/10.1177/0956797611430953

Kabadayi, C., Bobrowicz, K., \& Osvath, M. (2018). The detour paradigm in animal cognition. Animal Cognition, 21(1), 21-35. https://doi.org/10.1007/s10071-017-1152-0

Karp, N. A., Speak, A. O., White, J. K., Adams, D. J., Hrabé de Angelis, M., Hérault, Y., \& Mott, R. F. (2014). Impact of temporal variation on design and analysis of mouse knockout phenotyping studies. PloS One, 9(10), e111239. https://doi.org/10.1371/journal.pone.0111239

Kelley, L. A., \& Clayton, N. S. (2017). California scrub-jays reduce visual cues available to potential pilferers by matching food colour to caching substrate. Biology Letters, 13(7), 20170242. https://doi.org/10.1098/rsbl.2017.0242

Klein, R. A., Vianello, M., Hasselman, F., Adams, B. G., Adams, R. B., Alper, S., ... Nosek, B. A. (2018). Many Labs 2: Investigating Variation in Replicability Across Samples and Settings. Advances in Methods and Practices in Psychological Science, 1(4), 443-490.

https://doi.org/10.1177/2515245918810225 
Lazic, S. E., Clarke-Williams, C. J., \& Munafò, M. R. (2018). What exactly is ' $N$ ' in cell culture and animal experiments? PLOS Biology, 16(4), e2005282.

https://doi.org/10.1371/journal.pbio.2005282

Little, D. R., \& Smith, P. L. (2018). Replication is already mainstream: Lessons from small-N designs. Behavioral and Brain Sciences, 41. https://doi.org/10.1017/S0140525X18000766

Loken, E., \& Gelman, A. (2017). Measurement error and the replication crisis. Science, 355(6325), 584-585. https://doi.org/10.1126/science.aal3618

Maes, E., Boddez, Y., Alfei, J. M., Krypotos, A.-M., D’Hooge, R., De Houwer, J., \& Beckers, T. (2016). The elusive nature of the blocking effect: 15 failures to replicate. Journal of Experimental Psychology. General, 145(9), e49-71. https://doi.org/10.1037/xge0000200

McShane, B. B., \& Gal, D. (2016). Blinding Us to the Obvious? The Effect of Statistical Training on the Evaluation of Evidence. Management Science, 62(6), 1707-1718. https://doi.org/10.1287/mnsc.2015.2212

McShane, B. B., Gal, D., Gelman, A., Robert, C., \& Tackett, J. L. (2019). Abandon Statistical Significance. The American Statistician, 73(sup1), 235-245. https://doi.org/10.1080/00031305.2018.1527253

Morey, R. D. (2019, January 29). Why you shouldn't say "this study is underpowered." Retrieved September 22, 2019, from Medium website: https://towardsdatascience.com/why-youshouldnt-say-this-study-is-underpowered-627f002ddf35

Morey, R., \& Lakens, D. (2016). Why Most Of Psychology Is Statistically Unfalsifiable. https://doi.org/10.5281/zenodo.838685

Nosek, B. A., Ebersole, C. R., DeHaven, A. C., \& Mellor, D. T. (2018). The preregistration revolution. Proceedings of the National Academy of Sciences, 115(11), 2600-2606. https://doi.org/10.1073/pnas.1708274114 
Open Science Collaboration, O. S. (2015). PSYCHOLOGY. Estimating the reproducibility of psychological science. Science (New York, N.Y.), 349(6251), aac4716. https://doi.org/10.1126/science.aac4716

Piper, S. K., Grittner, U., Rex, A., Riedel, N., Fischer, F., Nadon, R., ... Dirnagl, U. (2019). Exact replication: Foundation of science or game of chance? PLOS Biology, 17(4), e3000188. https://doi.org/10.1371/journal.pbio.3000188

Poulin-Dubois, D., Rakoczy, H., Burnside, K., Crivello, C., Dörrenberg, S., Edwards, K., ... Ruffman, T. (2018). Do infants understand false beliefs? We don't know yet - A commentary on Baillargeon, Buttelmann and Southgate's commentary. Cognitive Development, 48, 302-315. https://doi.org/10.1016/j.cogdev.2018.09.005

Povinelli, D. J. (2003). Folk physics for apes: The chimpanzee's theory of how the world works. Oxford ; New York: Oxford University Press.

Robitsek, R. J., Fortin, N. J., Koh, M. T., Gallagher, M., \& Eichenbaum, H. (2008). Cognitive Aging: A Common Decline of Episodic Recollection and Spatial Memory in Rats. Journal of Neuroscience, 28(36), 8945-8954. https://doi.org/10.1523/JNEUROSCI.1893-08.2008

Schimmack, U. (2012). The ironic effect of significant results on the credibility of multiple-study articles. Psychological Methods, 17(4), 551-566. https://doi.org/10.1037/a0029487 Shettleworth, S. J. (2009). The evolution of comparative cognition: Is the snark still a boojum? Behavioural Processes, 80(3), 210-217. https://doi.org/10.1016/j.beproc.2008.09.001 Simmons, J. P., Nelson, L. D., \& Simonsohn, U. (2011). False-Positive Psychology. Psychological Science, 22(11), 1359-1366. https://doi.org/10.1177/0956797611417632

Simons, D. J., Shoda, Y., \& Lindsay, D. S. (2017). Constraints on Generality (COG): A Proposed Addition to All Empirical Papers. Perspectives on Psychological Science, 12(6), 1123-1128. https://doi.org/10.1177/1745691617708630

Smith, P. L., \& Little, D. R. (2018). Small is beautiful: In defense of the small-N design. Psychonomic Bulletin \& Review, 25(6), 2083-2101. https://doi.org/10.3758/s13423-018-1451-8 
Sterling, T. D. (1959). Publication Decisions and their Possible Effects on Inferences Drawn from Tests of Significance-Or Vice Versa. Journal of the American Statistical Association, 54(285), 3034. https://doi.org/10.1080/01621459.1959.10501497

Stevens, J. R. (2017). Replicability and Reproducibility in Comparative Psychology. Frontiers in Psychology, 8, 862. https://doi.org/10.3389/fpsyg.2017.00862

Thom, J. M., \& Clayton, N. S. (2013). Re-caching by Western Scrub-Jays (Aphelocoma californica) Cannot Be Attributed to Stress. PLoS ONE, 8(1), e52936. https://doi.org/10.1371/journal.pone.0052936

Vazire, S. (2018). Implications of the Credibility Revolution for Productivity, Creativity, and Progress. Perspectives on Psychological Science, 13(4), 411-417. https://doi.org/10.1177/1745691617751884

Voelkl, B., Vogt, L., Sena, E. S., \& Würbel, H. (2018). Reproducibility of preclinical animal research improves with heterogeneity of study samples. PLOS Biology, 16(2), e2003693. https://doi.org/10.1371/journal.pbio.2003693

Voelkl, B., \& Würbel, H. (2016). Reproducibility Crisis: Are We Ignoring Reaction Norms? Trends in Pharmacological Sciences, 37(7), 509-510. https://doi.org/10.1016/j.tips.2016.05.003

Voelkl, B., \& Würbel, H. (2019). A Reaction Norm Perspective on Reproducibility [Preprint]. https://doi.org/10.1101/510941

Wagenmakers, E.-J., Wetzels, R., Borsboom, D., \& van der Maas, H. L. J. (2011). Why psychologists must change the way they analyze their data: The case of psi: Comment on Bem (2011). Journal of Personality and Social Psychology, 100(3), 426-432. https://doi.org/10.1037/a0022790

Zwaan, R. A., Pecher, D., Paolacci, G., Bouwmeester, S., Verkoeijen, P., Dijkstra, K., \& Zeelenberg, R. (2018). Participant Nonnaiveté and the reproducibility of cognitive psychology. Psychonomic Bulletin \& Review, 25(5), 1968-1972. https://doi.org/10.3758/s13423-017-1348-y 


\section{Appendix A - Simulation Study}

The code for the simulations is available at: the code is available at https://github.com/BGFarrar/Pvalue-simulations/blob/master/CCreplicationsV1.R).

Data were simulated from two normal distributions for each of the four sets of simulations:

$\begin{array}{ccc}\text { Power } & \text { Population } 1 & \text { Population } 2 \\ 80 \% & X \sim N(50,5) & X \sim N(55.78,5) \\ 50 \% & X \sim N(50,5) & X \sim N(53.82,5) \\ 20 \% & X \sim N(50,5) & X \sim N(51.87,5) \\ 5 \% & X \sim N(50,5) & X \sim N(50,5)\end{array}$

The difference between Population 1 and Population 2 was calculated in order to give the desired power for a one-tailed two sample t-test with $n=10$ per group.

10,000 samples were then taken from each Population and compared to each other, and the $p$-values and mean difference between each sample recorded. The proportion of $p$-values under 0.05 was calculated, and the mean difference between samples associated with these $p$-values was compared to the mean difference across all samples to calculate the unstandarised effect size inflation.

Next, the expected number of exact replication studies that produced a significant result in the same direction as the original was calculated by multiplying the number of significant results from the simulation by the power of test again, and this was performed for a range of $p$ values (Table 2 ), as well as overall.

Finally, although not included in the manuscript, the exact replication studies were also simulated predictably, this was consistent with the mathematical derivation, and the p-value distributions of the original published studies and the replication studies are given below in Figure 1. 

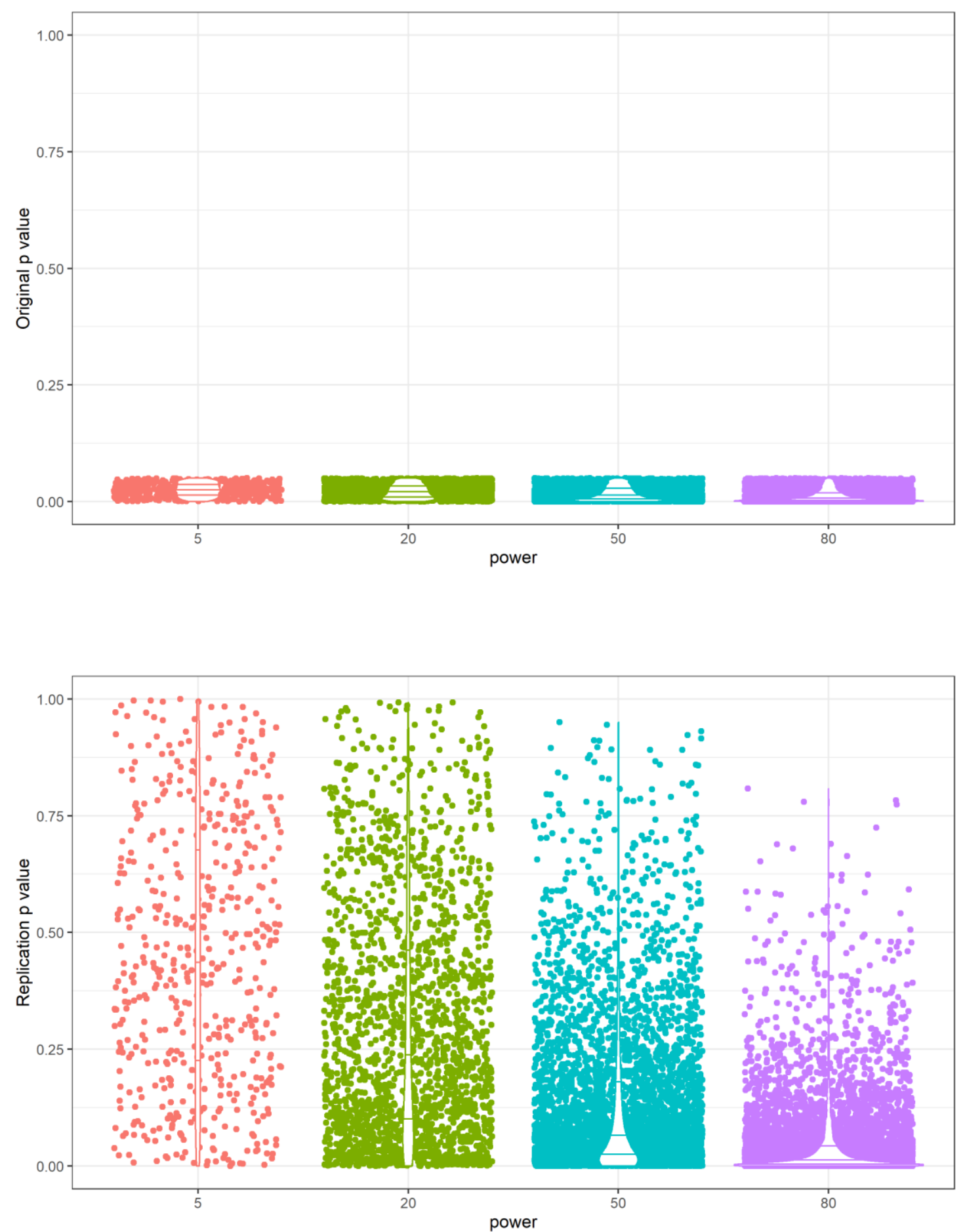

Figure 1: The p-value distributions of the original significant studies, by power (top), and the p-value distribution of their exact replication studies by power (bottom). Violin plots are overlaid displaying the range and quantiles 25,50 and 75. 